Viewpoint

\title{
Angry, Hungry T-Cells: How Are T-Cell Responses Induced in Low Nutrient Conditions?
}

\section{Francesco Nicoli}

Department of Chemical and Pharmaceutical Sciences, University of Ferrara, Ferrara, 44121, Italy; Email: nclfnc1@unife.it

\begin{abstract}
Upon activation, T-cells increase the uptake of glucose and glutamine to build the constituents of proliferating effectors. However, tumor and infected cells compete for the same nutrients. Several observations are consistently indicating that activated T-cells overcome this situation by engaging catabolic pathways. Here I discuss how these observations are reconciled with T-cells' need of anabolic processes during activation.
\end{abstract}

KEYWORDS: fatty acid oxidation; autophagy; AMPK; nutrient starvation; nutrient deficiency; T-cells

According to current paradigms, resting T-cells, once activated, shift from a catabolic to an anabolic metabolism which is necessary to sustain their growth, proliferation and effector functions. This energetic switch is controlled by mTOR, whose activation leads to induction of downstream processes including glutaminolysis and aerobic glycolysis-two pathways that provide intermediates needed for the biosynthesis of new nucleotides, amino acids and fatty acids (FA) for proliferating T-cells. To fuel this biosynthetic machinery, T-cells cannot just rely on internal nutrient stores. For instance, upon T-cell activation, new FAs are supplied for the cell not only via upregulation of FA synthesis (FAS) and lysis of triacylglycerols, but also by the cell's increased uptake of extracellular FAs [1,2]. Similarly, both glucose and glutamine intake are induced upon TCR triggering.

However, T-cell responses are deeply influenced by both the organism nutritional status and the local microenvironment. Protective immunity is indeed disrupted in both undernourished and overnourished individuals due to changes in circulating hormones and metabolites [3-5], a phenomenon exacerbated by the infections themselves, with further negative consequences on disease control [6,7]. In addition, T-cell responses take place at effector sites usually limited in nutrients [8,9]. For instance, infections often alter levels of certain metabolites in infected cells, in other immune cells interacting with lymphocytes or even systemically [7,10-14]. Additionally, at both infection and tumor sites, a huge competition for glucose and glutamine exists [8], and a high glycolytic rate in cancer cells has been shown to correlate with the low effector functions of intratumor T-cells [15]. The tumor micro- 
environment is also poor of co-stimulatory molecules, further preventing the metabolic switch in activated T-cells [16]. T-cells have shown to cope with these situations by shifting from glycolysis to glutaminolysis [14] and vice versa [17]. Conversely, tumor cells may not share the same metabolic plasticity. For instance, glycolysis inhibition blocks lactate secretion by tumor cells and augments T-cell infiltration and effector functions as well the effectiveness of checkpoint inhibitors [18]. Effector T-cells react to glucose withdrawal by boosting glutamine metabolism for ATP generation-a process controlled by the adenosine monophosphateactivated protein kinase (AMPK), whose activity is regulated by the AMP/ATP ratio [14]. Similarly, while glutaminolysis blocking disables tumor cells, it may improve T-cell functionality [17] by prompting the usage of FAs and glucose metabolism derivatives to fuel the tricarboxylic acid cycle and generate ATP [17]. High ATP levels will then down-modulate the AMPK activity [17] finally enabling the initiation of anabolic processes (which are inhibited by AMPK). Therefore, T cells activated in low nutrient conditions seem to alternate cycles of ATP generation (supported by AMPK) with cycles of anabolic processes (requiring AMPK inactivation).

Calorie restriction (CR) represents an interesting model to study T-cell behavior in low nutrient settings, as it enhances the responsiveness of $\mathrm{T}$ cells and reduces their terminal differentiation. In addition, CR induces autophagy $[19,20]$ and prompts T-cells to migrate into the bone marrow (BM), where they survive in a lipid-rich environment [21-24]. As CR affects both metabolite [25] and hormone [21] levels, the exact contribution of each single factor is difficult to define. Nevertheless, lipid usage and autophagy seem to be important pathways exploited by T-cells under CR. Notably, the induction of autophagy and lipid catabolism in activated Tcells may seem counter-intuitive, as both pathways are inhibited by mTOR. Autophagy induction is crucial for the survival of cells (including lymphocytes) during starvation or hypoxia [26-28] and for the maintenance of memory T-cells [26,27,29]. However, some recent publications have also described a role for autophagy in the stimulation of early-differentiated CD8 ${ }^{+}$T-cells, where it is induced simultaneously with mTOR triggering [30,31]. More than that, mTOR activity itself seems supported by autophagosomes [32]. In this respect, an interesting observation comes from the comparison of naïve with central memory Tcells. Both subsets reside in secondary lymphoid organs and have minimal energetic requirements. However, naïve T-cells display a lower basal nutrient uptake and thus suffer, de facto, from a limited access to nutrients. Nonetheless, they can compensate for this disadvantage by upregulating both mTOR and autophagy to levels higher than those of central memory T-cells [31]. Therefore, autophagy seems to provide additional energy to T-cells activated during nutrient deficiency. Similarly, FAO is considered the central energy source for resting T-cells [33], while TCR stimulation induces its opposite pathway, FAS. However, the induction of FAO during the priming of naïve $\mathrm{CD} 8^{+} \mathrm{T}$-cell has been shown 
to boost the functionality of new effectors without altering their proliferation rate [31], especially during nutrient deficiency within tumors [34-36]. Indeed, it has been demonstrated in both tumor and persistent infection models that T-cells activated in a glucose-deprived and hypoxic microenvironment show enhanced expression of inhibitory checkpoints $[34,37]$ associated with a reduced glycolysis and compensated by an increased FAO [34,38]. While the use of immune checkpoint blockers may in part reprogram T-cell metabolism [37,38], the simultaneous activation of FAO (through drugs) helps T-cells to overcome their reliance on glucose and improves their effector functions and the effectiveness of anti-PD-1 therapy [34-36]. These pieces of evidence show that metabolic pathways generally used by resting T-cells may be important upon activation to overcome poor nutrient availability. Nonetheless, it is unclear how proliferating T-cells, which exploit an mTOR-dependent anabolic metabolism, may be sustained by autophagy and FAO (which are rather inhibited by mTOR). The answer may derive in part from the observation of the bioenergetic features of resting memory T-cells. Indeed, despite the fact that they are considered highly reliant on FAO, their FA uptake is usually low. Quiescent T-cells are thought to themselves synthesize the FAs that will then be oxidized, alternating FAS and FAO [39]. FAS utilizes ATP, increasing the AMP/ATP ratio and inducing AMPK activity. AMPK then inhibits FAS and stimulates FAO, which generates ATP. This decreases the AMP/ATP ratio, switching off AMPK so that FAS may begin again, We cannot exclude that a similar cycle may occur during nutrient deprivation and that, in this way, AMPK may also control the mTOR/autophagy balance (e.g., alternating cycles of AMPK and mTOR activity).

In conclusion, several independent observations highlight that T-cells may meet their energetic demand during nutrient deficiency by using AMPK-controlled pathways such as autophagy and FAO [40]. However, to reconcile their metabolic requirements and growth needs, T-cells may be forced to alternate catabolic and anabolic processes in order to make up for the lack of biomolecules and construct the building blocks of proliferating cells, respectively. To finally translate immunometabolic studies into clinical practices, it will be crucial to finely dissect the kinetics of the different metabolic pathways engaged by T-cells in low nutrient settings (e.g., during infections, in tissues, during systemic metabolic alterations). Indeed, so far it is not yet clear whether the shift toward catabolic processes, enforced in nutrient-deprived settings, should be favored, to limit the competition for glucose [34-36], or should be rather counteracted, to improve T-cell glycolytic rate [41,42]. In addition, we are still far from knowing how different T-cell subpopulations react to poor biomolecule availability, and this constitutes a great limitation considering the huge amount of conditions, such as infections and age, that unbalance the proportion of early and late differentiated T-cell subsets [43-46]. It is therefore important that, when considering the use of molecules capable of affecting T-cell metabolism, such 
immunotherapeutic strategies should not desynchronize the bioenergetic processes of lymphocytes and thus undermine their capability to overcome nutrient deprivation.

\section{ACKNOWLEDGEMENTS}

I would like to thank Justin John Frere (Department of Microbiology, Icahn School of Medicine at Mount Sinai, New York, New York, USA) for fruitful discussion and editorial assistance.

\section{REFERENCES}

1. Dowell P, Otto TC, Adi S, Lane MD. Convergence of peroxisome proliferatoractivated receptor gamma and Foxo1 signaling pathways. J Biol Chem. 2003;278(46):45485-91.

2. Angela M, Endo Y, Asou HK, Yamamoto T, Tumes DJ, Tokuyama H, et al. Fatty acid metabolic reprogramming via mTOR-mediated inductions of PPARgamma directs early activation of T cells. Nat Commun. 2016;7:13683.

3. Alwarawrah Y, Kiernan K, MacIver NJ. Changes in Nutritional Status Impact Immune Cell Metabolism and Function. Front Immunol. 2018;9:1055.

4. He T, Xu C, Krampe N, Dillon SM, Sette P, Falwell E, et al. High-fat diet exacerbates SIV pathogenesis and accelerates disease progression. J Clin Invest. 2019;129(12):5474-88.

5. Christ A, Lauterbach M, Latz E. Western Diet and the Immune System: An Inflammatory Connection. Immunity. 2019;51(5):794-811.

6. Palmer CS, Palchaudhuri R, Albargy H, Abdel-Mohsen M, Crowe SM. Exploiting immune cell metabolic machinery for functional HIV cure and the prevention of inflammaging. F1000Res. 2018;7:125.

7. Palmer CS, Henstridge DC, Yu D, Singh A, Balderson B, Duette G, et al. Emerging Role and Characterization of Immunometabolism: Relevance to HIV Pathogenesis, Serious Non-AIDS Events, and a Cure. J Immunol. 2016;196(11):4437-44.

8. Kedia-Mehta N, Finlay DK. Competition for nutrients and its role in controlling immune responses. Nat Commun. 2019;10(1):2123.

9. Yang K, Chi H. AMPK helps T cells survive nutrient starvation. Immunity. 2015;42(1):4-6.

10. Gonzalez Plaza JJ, Hulak N, Kausova G, Zhumadilov Z, Akilzhanova A. Role of metabolism during viral infections, and crosstalk with the innate immune system. Intractable Rare Dis Res. 2016;5(2):90-6.

11. Zhang L, Romero P. Metabolic Control of CD8(+) T Cell Fate Decisions and Antitumor Immunity. Trends Mol Med. 2018;24(1):30-48.

12. Nicoli F, Paul S, Appay V. Harnessing the Induction of $\mathrm{CD}^{+} \mathrm{T}$-Cell Responses Through Metabolic Regulation by Pathogen-Recognition-Receptor Triggering in Antigen Presenting Cells. Front Immunol. 2018;9:2372. doi: 10.3389/fimmu.2018.02372

13. Telatin V, Nicoli F, Frasson C, Menegotto N, Barbaro F, Castelli E, et al. In chronic hepatitis $\mathrm{C}$ infection, myeloid-derived suppressor cell accumulation 
and $\mathrm{T}$ cell dysfunctions revert partially and late after successful direct-acting antiviral treatment. Front Cell Infect Microbiol. 2019;9:190.

14. Blagih J, Coulombe F, Vincent EE, Dupuy F, Galicia-Vazquez G, Yurchenko E, et al. The energy sensor AMPK regulates $\mathrm{T}$ cell metabolic adaptation and effector responses in vivo. Immunity. 2015;42(1):41-54.

15. Ho PC, Bihuniak JD, Macintyre AN, Staron M, Liu X, Amezquita R, et al. Phosphoenolpyruvate Is a Metabolic Checkpoint of Anti-tumor $\mathrm{T}$ Cell Responses. Cell. 2015;162(6):1217-28.

16. Menk AV, Scharping NE, Rivadeneira DB, Calderon MJ, Watson MJ, Dunstane $\mathrm{D}$, et al. 4-1BB costimulation induces $\mathrm{T}$ cell mitochondrial function and biogenesis enabling cancer immunotherapeutic responses. J Exp Med. 2018;215(4):1091-100.

17. Leone RD, Zhao L, Englert JM, Sun IM, Oh MH, Sun IH, et al. Glutamine blockade induces divergent metabolic programs to overcome tumor immune evasion. Science. 2019;366(6468):1013-21. doi: 10.1126/science.aav2588

18. Renner K, Bruss C, Schnell A, Koehl G, Becker HM, Fante M, et al. Restricting Glycolysis Preserves T Cell Effector Functions and Augments Checkpoint Therapy. Cell Rep. 2019;29(1):135-50.e9.

19. White MJ, Beaver CM, Goodier MR, Bottomley C, Nielsen CM, Wolf AF, et al. Calorie Restriction Attenuates Terminal Differentiation of Immune Cells. Front Immunol. 2016;7:667.

20. Bagherniya M, Butler AE, Barreto GE, Sahebkar A. The effect of fasting or calorie restriction on autophagy induction: A review of the literature. Ageing Res Rev. 2018;47:183-97.

21. Collins N, Han SJ, Enamorado M, Link VM, Huang B, Moseman EA, et al. The Bone Marrow Protects and Optimizes Immunological Memory during Dietary Restriction. Cell. 2019;178(5):1088-101 e15.

22. Shushimita S, de Bruijn MJ, de Bruin RW, IJzermans IJ, Hendriks RW, Dor FJ. Dietary restriction and fasting arrest $\mathrm{B}$ and $\mathrm{T}$ cell development and increase mature B and T cell numbers in bone marrow. PLoS One. 2014;9(2):e87772.

23. Contreras NA, Fontana L, Tosti V, Nikolich-Zugich J. Calorie restriction induces reversible lymphopenia and lymphoid organ atrophy due to cell redistribution. Geroscience. 2018;40(3):279-91.

24. Ahmed T, Das SK, Golden JK, Saltzman E, Roberts SB, Meydani SN. Calorie restriction enhances T-cell-mediated immune response in adult overweight men and women. J Gerontol A. 2009;64(11):1107-13.

25. Matyi S, Jackson J, Garrett K, Deepa SS, Unnikrishnan A. The effect of different levels of dietary restriction on glucose homeostasis and metabolic memory. Geroscience. 2018;40(2):139-49.

26. Botbol Y, Guerrero-Ros I, Macian F. Key roles of autophagy in regulating T-cell function. Eur J Immunol. 2016;46(6):1326-34.

27. Merkley SD, Chock CJ, Yang XO, Harris J, Castillo EF. Modulating T Cell Responses via Autophagy: The Intrinsic Influence Controlling the Function of Both Antigen-Presenting Cells and T Cells. Front Immunol. 2018;9:2914.

28. Singh R, Cuervo AM. Autophagy in the cellular energetic balance. Cell Metab. 2011;13(5):495-504. 
29. Xu X, Araki K, Li S, Han JH, Ye L, Tan WG, et al. Autophagy is essential for effector CD8(+) $\mathrm{T}$ cell survival and memory formation. Nat Immunol. 2014;15(12):1152-61.

30. Arnold CR, Pritz T, Brunner S, Knabb C, Salvenmoser W, Holzwarth B, et al. T cell receptor-mediated activation is a potent inducer of macroautophagy in human CD8(+)CD28(+) T cells but not in CD8(+)CD28(-) T cells. Exp Gerontol. 2014;54:75-83.

31. Nicoli F, Papagno L, Frere JJ, Cabral-Piccin MP, Clave E, Gostick E, et al. Naive CD8(+) T-Cells Engage a Versatile Metabolic Program Upon Activation in Humans and Differ Energetically From Memory CD8(+) T-Cells. Front Immunol. 2018;9:2736.

32. Whang MI, Tavares RM, Benjamin DI, Kattah MG, Advincula R, Nomura DK, et al. The Ubiquitin Binding Protein TAX1BP1 Mediates Autophagasome Induction and the Metabolic Transition of Activated T Cells. Immunity. 2017;46(3):405-20.

33. O’Sullivan D, van der Windt GJ, Huang SC, Curtis JD, Chang CH, Buck MD, et al. Memory CD8(+) T cells use cell-intrinsic lipolysis to support the metabolic programming necessary for development. Immunity. 2014;41(1):75-88.

34. Zhang Y, Kurupati R, Liu L, Zhou XY, Zhang G, Hudaihed A, et al. Enhancing $\mathrm{CD}^{+} \mathrm{T}$ Cell Fatty Acid Catabolism within a Metabolically Challenging Tumor Microenvironment Increases the Efficacy of Melanoma Immunotherapy. Cancer Cell. 2017;32(3):377-91.e9.

35. Chowdhury PS, Chamoto K, Honjo T. Combination therapy strategies for improving PD-1 blockade efficacy: a new era in cancer immunotherapy. J Intern Med. 2018;283(2):110-20.

36. Chowdhury PS, Chamoto K, Kumar A, Honjo T. PPAR-Induced Fatty Acid Oxidation in T Cells Increases the Number of Tumor-Reactive CD8(+) T Cells and Facilitates Anti-PD-1 Therapy. Cancer Immunol Res. 2018;6(11):1375-87. doi: 10.1158/2326-6066.CIR-18-0095

37. Chang CH, Qiu J, O’Sullivan D, Buck MD, Noguchi T, Curtis JD, et al. Metabolic Competition in the Tumor Microenvironment Is a Driver of Cancer Progression. Cell. 2015;162(6):1229-41.

38. Bengsch B, Johnson AL, Kurachi M, Odorizzi PM, Pauken KE, Attanasio J, et al. Bioenergetic Insufficiencies Due to Metabolic Alterations Regulated by the Inhibitory Receptor PD-1 Are an Early Driver of CD8(+) T Cell Exhaustion. Immunity. 2016;45(2):358-73.

39. Lochner M, Berod L, Sparwasser T. Fatty acid metabolism in the regulation of T cell function. Trends Immunol. 2015;36(2):81-91.

40. Pearce EL, Walsh MC, Cejas PJ, Harms GM, Shen H, Wang LS, et al. Enhancing CD8 T-cell memory by modulating fatty acid metabolism. Nature. 2009;460(7251):103-7.

41. Zhang C, Yue C, Herrmann A, Song J, Egelston C, Wang T, et al. STAT3 Activation-Induced Fatty Acid Oxidation in CD8(+) T Effector Cells Is Critical for Obesity-Promoted Breast Tumor Growth. Cell Metab. 2019. doi: 10.1016/j.cmet.2019.10.013 
42. DeVorkin L, Pavey N, Carleton G, Comber A, Ho C, Lim J, et al. Autophagy Regulation of Metabolism Is Required for CD8(+) T Cell Anti-tumor Immunity. Cell Rep. 2019;27(2):502-13.e5.

43. Appay V, Dunbar PR, Callan M, Klenerman P, Gillespie GM, Papagno L, et al. Memory CD8+ T cells vary in differentiation phenotype in different persistent virus infections. Nat Med. 2002;8(4):379-85.

44. Alanio C, Nicoli F, Sultanik P, Flecken T, Perot B, Duffy D, et al. Bystander hyperactivation of preimmune CD8+ T cells in chronic HCV patients. Elife. 2015;4:e07916. doi: 10.7554/eLife.07916

45. Nicoli F, Chachage M, Clowes P, Bauer A, Kowour D, Ensoli B, et al. Association between different anti-Tat antibody isotypes and HIV disease progression: data from an African cohort. BMC Infect Dis. 2016;16:344.

46. Briceno O, Lissina A, Wanke K, Afonso G, von Braun A, Ragon K, et al. Reduced naive CD8(+) T-cell priming efficacy in elderly adults. Aging Cell. 2016;15(1):14-21.

How to cite this article:

Nicoli F. Angry, Hungry T-Cells: How Are T-Cell Responses Induced in Low Nutrient Conditions? Immunometabolism. 2020;2(1):e200004. https://doi.org/10.20900/immunometab20200004 